\title{
Controlling spurious diapycnal mixing in eddy-resolving height-coordinate ocean models - Insights from virtual deliberate tracer release experiments
}

\author{
Chris Hill*, David Ferreira, Jean-Michel Campin, John Marshall, Ryan Abernathey, Nicolas Barrier \\ Department of Earth, Atmospheric and Planetary Science, Massachusetts Institute of Technology, Cambridge, MA 02139, USA
}

\section{A R T I C L E I N F O}

\section{Article history:}

Available online 15 December 2011

\section{Keywords}

Ocean modeling

Diapyncnal mixing

Numerical mixing

Advection

Southern ocean

Adiabatic

\begin{abstract}
A B S T R A C T
A perceived limitation of z-coordinate models associated with spurious diapycnal mixing in eddying, frontal flow, can be readily addressed through appropriate attention to the tracer advection schemes employed. It is demonstrated that tracer advection schemes developed by Prather and collaborators for application in the stratosphere, greatly improve the fidelity of eddying flows, reducing levels of spurious diapycnal mixing to below those directly measured in field experiments, $\sim 1 \times 10^{-5} \mathrm{~m}^{2} \mathrm{~s}^{-1}$. This approach yields a model in which geostrophic eddies are quasi-adiabatic in the ocean interior, so that the residual-mean overturning circulation aligns almost perfectly with density contours. A reentrant channel configuration of the MIT General Circulation Model, that approximates the Antarctic Circumpolar Current, is used to examine these issues. Virtual analogs of ocean deliberate tracer release field experiments reinforce our conclusion, producing passive tracer solutions that parallel field experiments remarkably well.
\end{abstract}

(c) 2011 Elsevier Ltd. All rights reserved.

\section{Introduction}

Observations and theory (Ledwell et al., 1993, 1998, 2011; Garrett and Munk, 1972) suggest that tracers in the ocean's thermocline are stirred and mixed overwhelmingly along isopycnal surfaces. Ocean eddies distort and filament contours of tracers, such that along-isopycnal mixing of order $\sim 10^{3} \mathrm{~m}^{2} \mathrm{~s}^{-1}$ is typically observed (e.g. Abernathey et al., 2010). Diapycnal mixing on the other hand, which arises primarily through internal wave breaking, is observed to be many orders of magnitude smaller, $\sim 10^{-5} \mathrm{~m}^{2} \mathrm{~s}^{-1}$, away from surface and bottom boundaries. For example, Ledwell et al. $(1998,2011)$ estimated, from tracer release experiments, diapycnal mixing of $1.7 \pm 0.2 \times 10^{-5} \mathrm{~m}^{2} \mathrm{~s}^{-1}$ at $300 \mathrm{~m}$ depth in the North Atlantic "NATRE" experiment and $1.3 \pm 0.2 \times 10^{-5} \mathrm{~m}^{2} \mathrm{~s}^{-1}$ at $1500 \mathrm{~m}$ in the recent Southern Ocean "DIMES" experiment, while microstructure measurements indicate widespread values of $10^{-5} \mathrm{~m}^{2} \mathrm{~s}^{-1}$ above smooth abyssal plains in the South Atlantic (Polzin et al., 1997).

Asymmetry in isopycnal and diapycnal mixing rates can be readily imposed in numerical models which use isopycnal coordinates. However, it is a much greater challenge to faithfully reproduce this asymmetry in models that employ height, $z$ (or pressure $p$ ) in which the horizontal coordinate is parallel to geopotential surfaces and may, therefore, cross isopycnals. In particular, in strongly eddying, frontal flows in which isopycnal surfaces

\footnotetext{
* Corresponding author. Tel.: +1 617253 6430; fax: +1 6172534464

E-mail address: cnh@mit.edu (C. Hill).
}

become steeply inclined to the geopotential horizontal, the fundamental 'horizontal bias' of z-coordinate models can lead to spurious diapycnal mixing in which isopycnal processes become erroneously projected onto the cross-isopycnal direction. This was convincingly argued by Griffies et al. (2000) (hereafter GPHO0) who showed that the numerically generated diapycnal mixing rates associated with centered, second order advection schemes deployed in eddying regimes, can reach levels of $10^{-4} \mathrm{~m}^{2} \mathrm{~s}^{-1}$, an order of magnitude larger than values observed in the thermocline. Such spurious mixing is often used as an argument in favor of isopycnal coordinate approaches.

This paper examines how to reduce spurious, eddy-induced, diapycnal mixing in z-coordinate models. We find such mixing can be corrected through appropriate attention to numerical tracer advection schemes. In particular, adopting ideas from First and Second Order Moment (SOM) advection schemes (Prather, 1986; Russell and Lerner, 1981) markedly improves tracer advection properties of ocean models. These schemes have already been employed in an oceanographic context and exhibited encouraging properties (Merryfield and Holloway, 2003; Morales Maqueda and Holloway, 2006; Hofmann and Morales Maqueda, 2006; Tatebe and Hasumi, 2010). Those studies however explored the large scale circulation of the ocean (and associated sea-ice processes in Merryfield and Holloway, 2003) in a non-eddying regime - they did not examine the issues raised by GPHOO around eddy resolving simulation.

Here we examine tracer advection in an eddying numerical model of an idealized Antarctic Circumpolar Current (ACC). Our 
goal is to (1) evaluate the effective diapycnal mixing associated with SOM schemes and (2) determine whether SOM schemes can render a z-coordinate model in which characteristic ocean properties - weak interior diapycnal mixing and interior residual circulation aligned with isentropic surfaces - are accurately represented. To this end, we carry out numerical experiments that are virtual analogs of tracer release field experiments such as those undertaken by Ledwell and collaborators during the NATRE and DIMES campaigns. Such experiments permit the estimation of the effective diapycnal diffusion undergone by a passive tracer in a numerical model. Results are further compared to those of the "spin-down" method of GPHOO which can estimate effective diffusivities experienced by active tracers. We find that, through use of the SOM schemes, we are able to accurately reproduce, for the first time in a z-coordinate model of a highly turbulent flow, oceanographic field measurements of tracer release. This is achieved because rates of spurious mixing due to numerics become smaller than observed diapycnal mixing rates. Climate science implications of the ability of a z-coordinate model to sustain a quasi-adiabatic ocean interior in an eddying regime are further illustrated through computations of residual-mean meridional overturning circulation.

Our paper is set out as follows. In Section 2 the origin of spurious mixing in discrete numerical models is discussed, and a set of experiments used to evaluate levels of effective diapycnal mixing are described. The numerical experiments are derived from a common physical scenario, a wind and buoyancy forced reentrant channel, as employed by Abernathey et al. (2011). In this section we also outline the three different approaches used to evaluate diapycnal exchange. In Section 3, we use these approaches to assess the levels of spurious mixing associated with a variety of advection schemes. In Section 4 we summarize our thinking on the implications of our experiments for modeling an eddying ocean.

\section{Modeling tracer transport}

Tracer advection in a numerical Eulerian coordinate model inevitably introduces some artificial diffusion and dispersion. For a three-dimensional non-divergent flow field, $\vec{u}=(u, v, w)$, such as occurs in incompressible ocean flows, the advection equation of a (passive or active) tracer $\phi$ in a fixed, Eulerian, coordinate is:

$\frac{\partial \phi}{\partial t}=-u \frac{\partial \phi}{\partial x}-v \frac{\partial \phi}{\partial y}-w \frac{\partial \phi}{\partial z}$

Written as in Eq. (1) advection by a flow field does not mix, but in an Eulerian numerical discretization some mixing of tracer will arise. In the analysis that follows we examine the magnitude of effective diapycnal diffusivity associated with this mixing. The effective diapycnal mixing in this scenario will be a result of numerical effects not physical processes. Our interest is in whether and when, in a model, this term is below observed values in low mixing regions of the ocean interior. Following observational studies (and a number of previous numerical examinations), we quantify the degree of mixing by using measures of effective diapycnal diffusivity diagnosed numerically from simulation solutions. These effective diffusivities are spurious in the sense they derive from numerical terms not inherent in (1).

When discretized in space, the terms on the rhs of Eq. (1) must be approximated with bulk averages over a finite sized region, typically:

$\frac{\partial \bar{\phi}}{\partial t}=-\delta_{x}(\bar{U} \bar{\phi})-\delta_{y}(\bar{V} \bar{\phi})-\delta_{z}(\bar{W} \bar{\phi})$

where - denotes a bulk average field and $\delta$ a discrete approximation to the gradient operator in (1). The transport components $(\bar{U}, \bar{V}, \bar{W})$ are written upper case to indicate that they may be area weighted transports in the case of a model with variable grid spacing. The discretization details in evaluating (2) computationally lead to varying degrees of "spurious" (i.e. numerically induced) diffusion and dispersion in solutions to Eq. (1) (GPH00; Rood, 1987). This diffusion occurs along each model coordinate $x, y$ and $z$.

For accurate simulation, it is essential that spurious "numerical" diffusion in an advection scheme be as low as possible and certainly lower than values observed in the fluid being simulated. As mentioned in the introduction, interior ocean mixing is predominantly isopycnal. In a geopotential coordinate model the grid directions $x, y$ and $z$ will not be aligned with isopycnals and so numerical diffusion arising from (2) can project in the diapycnal direction. As emphasized by GPH00, this is particularly true in an eddying regime where sharply tilted isopycnals naturally emerge in the ocean interior as part of the flow solution.

\subsection{Model configuration}

We conduct a series of numerical experiments with the MITgcm (Marshall et al., 1997a,b) to address the performance of various numerical advection schemes for solving Eq. (2). We study an "active" tracer, potential temperature which affects the density of the fluid, and a "passive" tracer, a dye that does not affect fluid density. Our model configuration is an idealized $\beta$-plane representation of the ACC. It consists of a zonally periodic channel of length $1000 \mathrm{~km}$ and width $2000 \mathrm{~km}$. The channel has a flat bottom and is $3 \mathrm{~km}$ deep. The surface wind stress is purely zonal (blowing eastward) with a magnitude varying sinusoidally across the channel. It peaks at $0.1 \mathrm{~N} \mathrm{~m}^{-2}$ in the middle of the channel and is zero at the northern and southern boundaries. A linear bottom drag is used to dissipate momentum in the bottom layer. The equation of state is assumed to be linear with a single state variable, $\theta$, representing potential temperature so that isotherms and isopycnals are equivalent. The thermal expansion coefficient is $2 \times 10^{-4} \mathrm{~K}^{-1}$. A prescribed (zonally symmetric) air-sea heat flux creates alternating regions of surface cooling and heating. Fig. 1 illustrates the configuration, showing a snapshot of the model for one particular experiment.

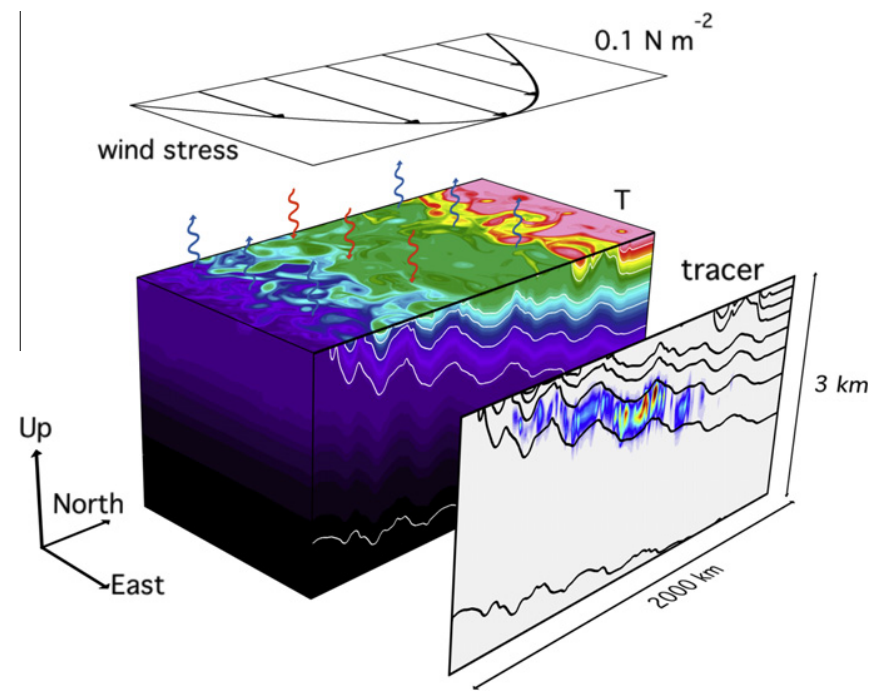

Fig. 1. Rentrant (zonally periodic) channel model. The $3 \mathrm{~d}$ rendition shows a snapshot of the temperature field in the $2000 \mathrm{~km}$ wide, $1000 \mathrm{~km}$ long and $3 \mathrm{~km}$ deep channel. The temperature ranges between 0 and $8{ }^{\circ} \mathrm{C}$. Wavy arrows indicate surface heat flux, blue and red are out of and into the ocean, respectively. The magnitude of the surface heat flux is $\pm 10 \mathrm{~W} \mathrm{~m}^{-2}$. The meridional profile of the zonal wind stress blowing over the channel is shown at the top. To the right are contours of the temperature for the zonal section at the limit of the domain. Overlaid in color is the tracer concentration for a tracer released into the flow. The experiment shown uses the SOM advection scheme. (For interpretation of the references in color in this figure legend, the reader is referred to the web version of this article.) 

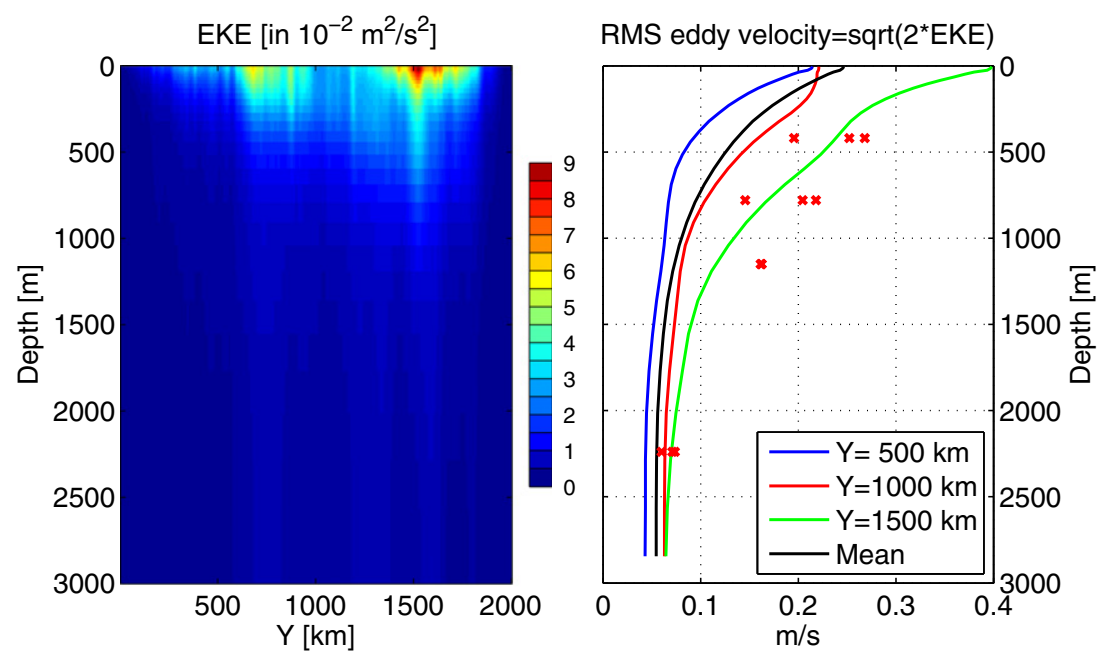

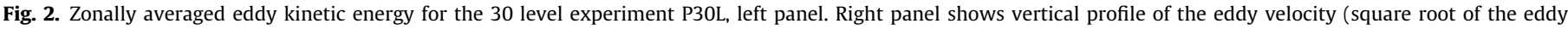

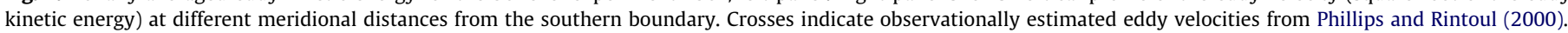

The prescribed forcing pattern drives a residual overturning with upwelling of dense water on the southern flank of an ACC-like current and subduction on its equatorial flank. On the northern boundary, temperature is relaxed to a prescribed profile within a $100 \mathrm{~km}$ wide equatorial flank sponge layer. The KPP mixed-layer scheme is used (Large et al., 1994). The horizontal grid spacing is $5 \mathrm{~km}$ for the bulk of the experiments we describe - and so reasonably resolves the first baroclinic deformation radius of approximately $20 \mathrm{~km}$. One additional experiment, using horizontal resolution of $2.5 \mathrm{~km}$ is carried out, to examine the impact of higher lateral resolution. An Adams-Bashforth time stepping scheme is used with a stabilization factor of 0.1 in the momentum equation and also for any tracer that uses the centered 2nd order advection scheme. For all the main experiments a fixed value biharmonic viscosity is used as momentum closure with a value of $9 \times 10^{8} \mathrm{~m}^{4} \mathrm{~s}^{-1}$. The only exception is the enhanced horizontal resolution experiment, with $2.5 \mathrm{~km}$ horizontal grid spacing. In that experiment biharmonic viscosity is reduced to $9 \times 10^{8} \mathrm{~m}^{4} \mathrm{~s}^{-1} \times \frac{1}{16}$. A limited test with double the viscosity in the $2.5 \mathrm{~km}$ resolution case showed relatively little sensitivity of the results presented here to viscosity in this range. Momentum advection is configured to use a second order scheme. We did not investigate the impact of alternate momentum advection formulations although this may also impact spurious mixing (Ilicak et al., in press).

We use two different vertical resolutions: in one configuration, three hundred $10 \mathrm{~m}$ thick levels are employed and in the other, a set of thirty levels with thicknesses ranging from $10 \mathrm{~m}$ at the surface to $280 \mathrm{~m}$ near the bottom. The reader is referred to Abernathey et al. (2011) for further details and analysis of the dynamics of the channel. As illustrated by a snap-shot of temperature (Fig. 1 (left) from one specific experiment, P30 - see below), the flow is strongly eddying. The temperature slice (Fig. 1 right) shows the deep density surfaces to the north rising and outcropping to the south in a manner that is reminiscent of the ACC. Color shading on the contour slice shows tracer concentration for a passive dye injection on an isopycnal surface (as in Ledwell et al. (1993) and similar experiments). In the experiment shown, the dye remains confined between isopycnal (isotherms in our case) surfaces, visual confirmation of low diapycnal mixing rates for this case. Fig. 2 shows the zonally averaged eddy kinetic energy for the channel. The left panel shows a depth versus meridional distance color map. The right panel shows curves of variation of RMS eddy velocity with depth at meridional distances of 500, 1000 and $1500 \mathrm{~km}$ together with observational estimates from moorings deployed in the core of the ACC south of Australia (Phillips and Rintoul, 2000).

\subsection{Numerical experiments}

Based on the numerical configuration outlined above, we spinup to statistical equilibrium six standard horizontal resolution and one enhanced horizontal resolution flow scenarios. In these seven experiments various advection schemes and vertical resolutions are used. Key details are outlined in Table 1.

Thirty-level experiments, denoted $\mathbf{3 0}$ in Table 1, illustrate the impact of advection schemes in a vertical grid configuration comparable to contemporary practices in large scale ocean modeling. For this configuration, we undertake experiments with four different advection approaches applied to temperature (the active tracer in our model); a centered second-order scheme (labelled C30); a 7th order one-step method, with monotonicity preserving limiter based on Daru and Tenaud (2004) (labelled S30); and a second-order moment (SOM) scheme based on Prather (1986) with and without flux limiting (applying the Prather (1986) limiter ${ }^{1}$ ) to enforce positive definite behavior. Experiments with the Prather (1986) limiter are labelled P30L, those without are labelled P30. Experiment $\mathbf{C 3 0}$ includes explicit horizontal and vertical background diffusivities to alleviate numerical dispersion. All other calculations have no explicit diffusivity term. A Prather (1986) based experiment with 300 levels (labelled P300) was carried out to provide a configuration that can be compared more directly to the NATRE and DIMES deliberate tracer release results. The $\mathbf{P 3 0 0}$ calculation also provides a benchmark comparison between effective diffusivities estimated from (1) the Ledwell et al. and (2) GPHOO methods with numerical differences minimized (see Appendix for details). This experiment is also compared with another 300 level experiment $\mathbf{S 3 0 0}$, a 300 level equivalent of the $\mathbf{S 3 0}$ experiment. The $\mathbf{S 3 0 0}$ experiment allows intercomparison between two different state-of-the-art advection schemes in the context of enhanced vertical resolution. The 300 level experiments are used to interpret small discrepancies between the diffusivities computed by the 2 diagnostic methods in the 30-level set-ups. In the 30-level case these discrepancies are partially due

\footnotetext{
1 The limiter used, denoted Lim $=1$ in Prather et al. (2008), limits any fluxes that would generate spurious negative values of tracer concentrations, as a result advected concentrations remain positive in tracer release experiments described in Section 2.4
} 
Table 1

Table of numerical experiments showing labels, vertical resolution, advection scheme and any explicit vertical and horizontal diffusivity (in $\mathrm{m}^{2} \mathrm{~s}^{-1}$ ). Advection and diffusion applies to temperature, the only active tracer. All experiments except P30_HR have a horizontal resolution of $5 \mathrm{~km}$, the P30_HR experiment has $2.5 \mathrm{~km}$ lateral resolution.

\begin{tabular}{llll}
\hline Label & \# of Levels & Advection & $K_{v}, K_{h}$ \\
\hline C30 & 30 & Centered 2nd order & $10^{-5}, 30$ \\
S30 & 30 & High-order, monotonicity preserving & None \\
P30 & 30 & Prather, no limiter & None \\
P30L & 30 & Prather, limiter & None \\
P30_HR & 30 & Prather & None \\
& & Enhanced horizontal resolution & \\
P300 & 300 & Prather, no limiter & None \\
S300 & 300 & High-order, monotonicity preserving & None \\
\hline
\end{tabular}

to approximations associated with applying the methods with a coarse vertical grid (again see the Appendix for examples).

Each of the flow scenarios is first spun up to a state of statistical equilibrium. From these equilibrated states, we then examine the qualitative and quantitative diapycnal mixing rates using (i) "spin down" experiments as in GPH00, (ii) numerical/"virtual" passive tracer release experiments for multiple different passive tracer advection schemes and (iii) residual-mean circulation calculations.

\subsection{Spin-down experiments}

In these experiments, we monitor the evolving temperature field after all externally imposed forcing is turned off. The isotherms slump adiabatically as the fluid relaxes towards a resting state. During the spin-down, the volume of water in a particular temperature class cannot change unless there is mixing present. Any effective diapycnal mixing can therefore be diagnosed by tracking changes in the volume of water, $V^{\theta}(t)$, in a specific temperature class, $\theta \pm \delta \theta$ as a function of time, $t$. The approach is discussed at length in GPHOO and illustrated in Fig. 3 for the case of a twodimensional $(x-z)$ set-up. The left panel shows a schematic of a system at a time $t$ with three temperature classes lying in an $x-z$ plane. The middle and right panels illustrate transformations that are the basis for computing effective diapycnal mixing. First, the fluid parcels are rearranged adiabatically into a stably stratified, horizontally layered state, as one would do to compute the minimum potential energy state. Second, the temperature is plotted against a pseudo-depth $z^{*}$ related to the area occupied by each temperature class (right panel). One can readily see that, in spindown experiments, the temperature profile $\theta\left(z^{*}, t\right)$ would be constant in time in the absence of diapycnal mixing (i.e. the area, or volume in 3d, of each class would be constant). GPH00 show that, by ascribing changes in $\theta\left(z^{*}, t\right)$ to an effective diapycnal diffusivity, $\kappa_{\text {eff }}^{\mathrm{G}}$, the relation

$\frac{\partial \theta\left(z^{*}, t\right)}{\partial t}=\frac{\partial}{\partial z^{*}}\left[\kappa_{\mathrm{eff}}^{\mathrm{G}}\left(z^{*}, t\right) \frac{\partial \theta\left(z^{*}, t\right)}{\partial z^{*}}\right]$

can be used to diagnose diapycnal mixing. Thus, using the time history of the numerical state in the spin-down experiment, $\kappa_{\text {eff }}^{\mathrm{G}}$ can be inferred. The approach applies equally well to more complex, three-dimensional, water mass distributions in which water mass volumes may be in disconnected patches and to any flat-bottomed three-dimensional domain. In our experiments, we report mean $\kappa_{\mathrm{eff}}^{\mathrm{G}}\left(z^{*}\right)$ values, which are monthly averages of a sequence of $\kappa_{\text {eff }}^{\mathrm{G}}\left(z^{*}, t\right)$ values, each computed by differencing successive samples of model state. The resulting $\kappa_{\text {eff }}^{\mathrm{G}}\left(z^{*}\right)$ is the average diapycnal mixing across a particular isotherm.

\subsection{Tracer release tests}

For each vertical resolution, 8 passive tracer release experiments are carried out. The eight (passive) tracers are advected by the same flow fields (taken from $\mathbf{P 3 0 0}$ and P30), but with eight different advection schemes as follows: (A1) SOM (no limiter); (A2) SOM with limiter; (A3) SOM (no limiter) with diffusion; (A4) centered 2nd order with diffusion; (A5) 2nd order with a "Superbee" (Roe, 1985) flux limiter; (A6) 3rd order direct space time (Hundsdorfer and Verwer, 2003); (A7) 3rd order direct space time with Sweby limiter (Sweby, 1984); and (A8) a 7th order one-step method, with monotonicity preserving limiter (Daru and Tenaud, 2004). When diffusion is employed with an advection scheme both explicit vertical and horizontal diffusivities are used - with values set to $10^{-5}$ and $30 \mathrm{~m}^{2} \mathrm{~s}^{-1}$, respectively. ${ }^{2}$ The flux limited advection schemes ensure that tracer values remain positive definite.

In these experiments a tracer is released into a localized patch in a spun up simulation - a numerical analog of a tracer release field experiment. The method to infer an effective vertical diffusivity (in units of $\mathrm{m}^{2} \mathrm{~s}^{-1}$ ) is a close analogy of the observational procedure used in field programs, as follows. The 3d tracer distribution is sampled at ten day intervals after injection. The tracer concentrations vary in space as they are stirred along sloping isotherms and diffused horizontally and vertically (explicitly and/ or numerically). In order to calculate an effective diffusivity, all vertical profiles are first plotted around a relative vertical coordinate centered on the target temperature of the release and then summed up into one single profile. If the effective diffusivity is uniform vertically, the reconstructed profile will assume a Gaussian shape whose half-width, $\sigma$, evolution in time is related to the effective diffusivity, (here denoted $\kappa_{\text {eff }}^{\mathrm{L}}$ to distinguish it from the spindown experiment computed diffusivity, $\kappa_{e f f}^{G}$ ), through the equation:

$\kappa_{\mathrm{eff}}^{\mathrm{L}}=\frac{1}{2} \frac{d \sigma^{2}}{d t}$

Values of $\kappa_{\text {eff }}^{\mathrm{L}}$, computed from a linear best fit of $\sigma^{2}(t)$ to (4), are given in Section 3. For more details, the reader is referred to Ledwell et al. $(1993,1998)$. A discussion of issues related to the implementation of this method in an ocean model is given in the Appendix.

\subsection{Residual-mean streamfunction}

The final diagnostic that we employ is an explicit computation of the residual-mean meridional overturning streamfunction $\psi_{\text {iso }}$. This is diagnosed by first computing, at each latitude $y$ and time $t$, the total meridional volume flux per temperature class, $M(y, \theta, t)$. The residual-mean circulation is then obtained by integrating in temperature and averaging over time:

$\psi_{\text {iso }}(y, \theta)=-{\overline{\int_{\theta_{\min }}^{\theta} M(y, \theta, t) d \theta}}^{t}$

where $\theta_{\min }$ is the minimum temperature and the overbar denotes a time average. As demonstrated by McIntosh and McDougall (1996), $\psi_{\text {iso }}$ is close to the Transformed-Eulerian-Mean used by Andrews and McIntyre (1976), Treguier et al. (1997) and Marshall and Radko (2003) among others. In our calculations, the temperature layers are chosen to be $0.2 \mathrm{~K}$ thick, the volume flux is computed at every time step and the averaging period is 5 years. This yields the overturning streamfunction in temperature coordinates shown in Section 3. Adiabatic interior residual circulation will appear as horizontal con-

\footnotetext{
2 The explicit horizontal diffusion used here is for numerical purposes, and should not be confused with the (considerably larger) effective isopycnal eddy diffusivity mentioned in the Introduction that results from eddy stirring.
} 


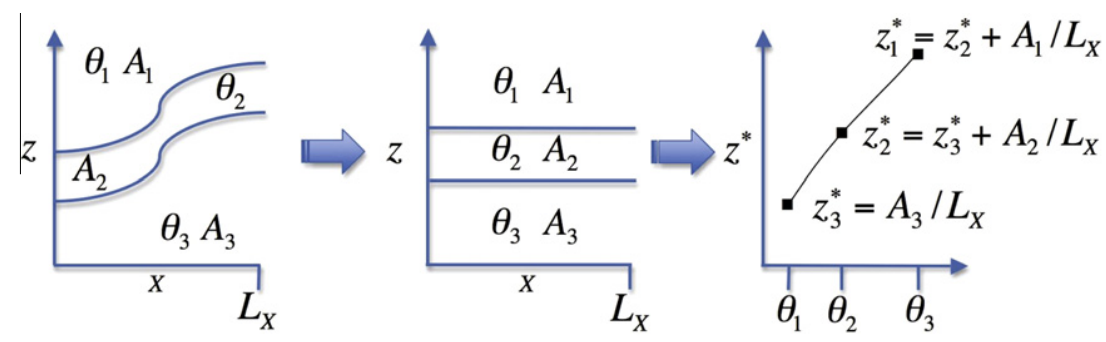

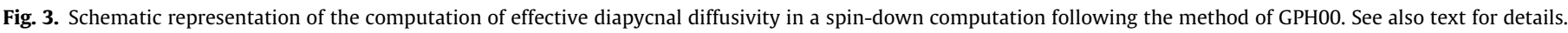

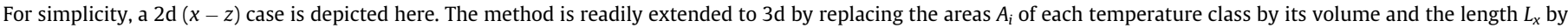
the area of the Channel $L_{x} \times L_{y}$.

tours in plots of $\psi_{\text {iso. }}$. This diagnostic does not result in a numerical measure of diapycnal exchange, but it illustrates qualitatively the large scale implications of diapycnal exchange.

\section{Results}

We now describe results from the experiments discussed in Section 2. We first present the 300 level reference experiment looking at GPHOO and passive tracer diagnostics of diapycnal mixing. We then examine the same measures in thirty level experiments. Finally we diagnose residual circulations in each experiment.

\subsection{0 level experiment}

We first present results from the 300 level SOM experiment, P300. Effective diffusivities, $\kappa_{\text {eff }}^{G}$, diagnosed using the GPH00 method are shown in Fig. 4. The effective diffusivities, as a function of $z^{*}$ (using the GPHOO convention that $z^{*}=0$ is the ocean bottom), over a 50 day spin-down are shown on the left panel with the timeaverage value in the right panel. Here, $\kappa_{\text {eff }}^{\mathrm{G}}$ is fairly constant over time, only a small decay is noticeable at high $z^{*} /$ temperature. The eddy field remains vigorous throughout the spin-down period.
Therefore, the estimated $\kappa_{\text {eff }}^{\mathrm{G}}$ should be representative of the eddying system. There is no explicit diapycnal diffusion prescribed in P300, and thus the $\kappa_{\text {eff }}^{G}$ estimated here is purely spurious. However, the values are well below the limit of $10^{-5} \mathrm{~m}^{2} \mathrm{~s}^{-1}$ everywhere except near the boundaries. In fact, on the deep isotherms $\left(z^{*}\right.$ between 500 and $1500 \mathrm{~m}$ ), the effective diffusivities are as low as $0.02 \times 10^{-5} \mathrm{~m}^{2} \mathrm{~s}^{-1}$. Somewhat larger values found above $z^{*}=1500 \mathrm{~m}$ correspond to isotherms which outcrop. As there is more filamenting and a stronger cascade to small scales near the surface, stronger temperature gradients arise at the grid scale and thus larger spurious mixing is expected. These relatively high values near the surface are of little concern, first because they remain below the $10^{-5} \mathrm{~m}^{2} \mathrm{~s}^{-1}$ threshold, but more importantly because, in the forced system (and in the real ocean), the surface layer is a place of high mixing due to turbulent mixed-layer dynamics. A 30 level experiment, P30_HR, with higher horizontal resolution (see Section 3.2) is consistent with this explanation.

Fig. 4 also shows comparable results for the $\mathbf{S 3 0 0}$ experiment, that uses advection described in Daru and Tenaud (2004). This advection algorithm is a high-order monotonicity preserving scheme. This experiment was carried out to examine an alternate state-of-the-art advection scheme to the SOM based computations. At depths that correspond to the deliberate tracer release results of Ledwell, the $\kappa_{\text {eff }}^{G}$ mixing diagnostic has a value of $\approx 10^{-5} \mathrm{~m}^{2} \mathrm{~s}^{-1}$. The

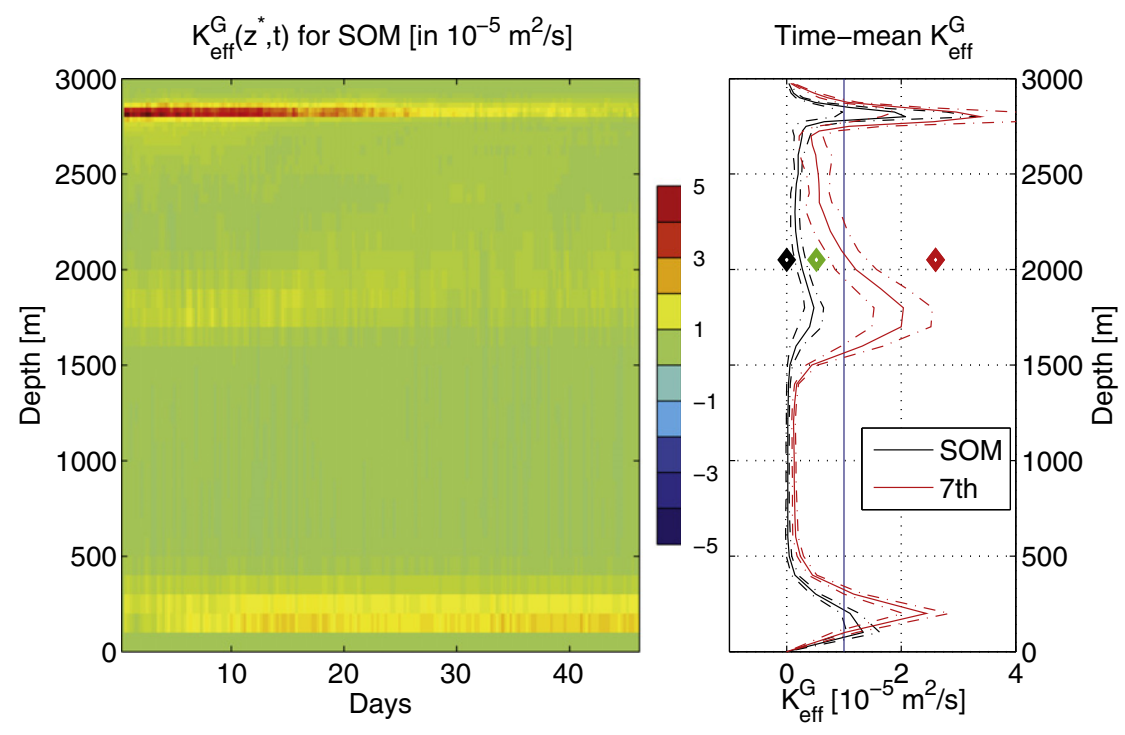

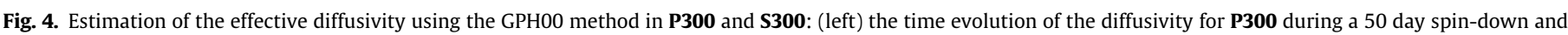

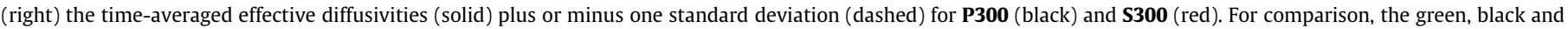

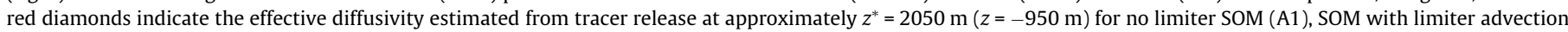

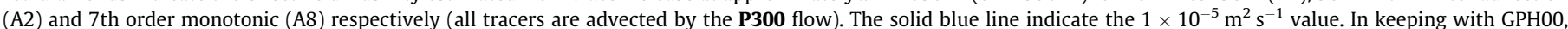

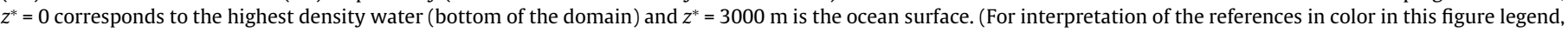
the reader is referred to the web version of this article.) 

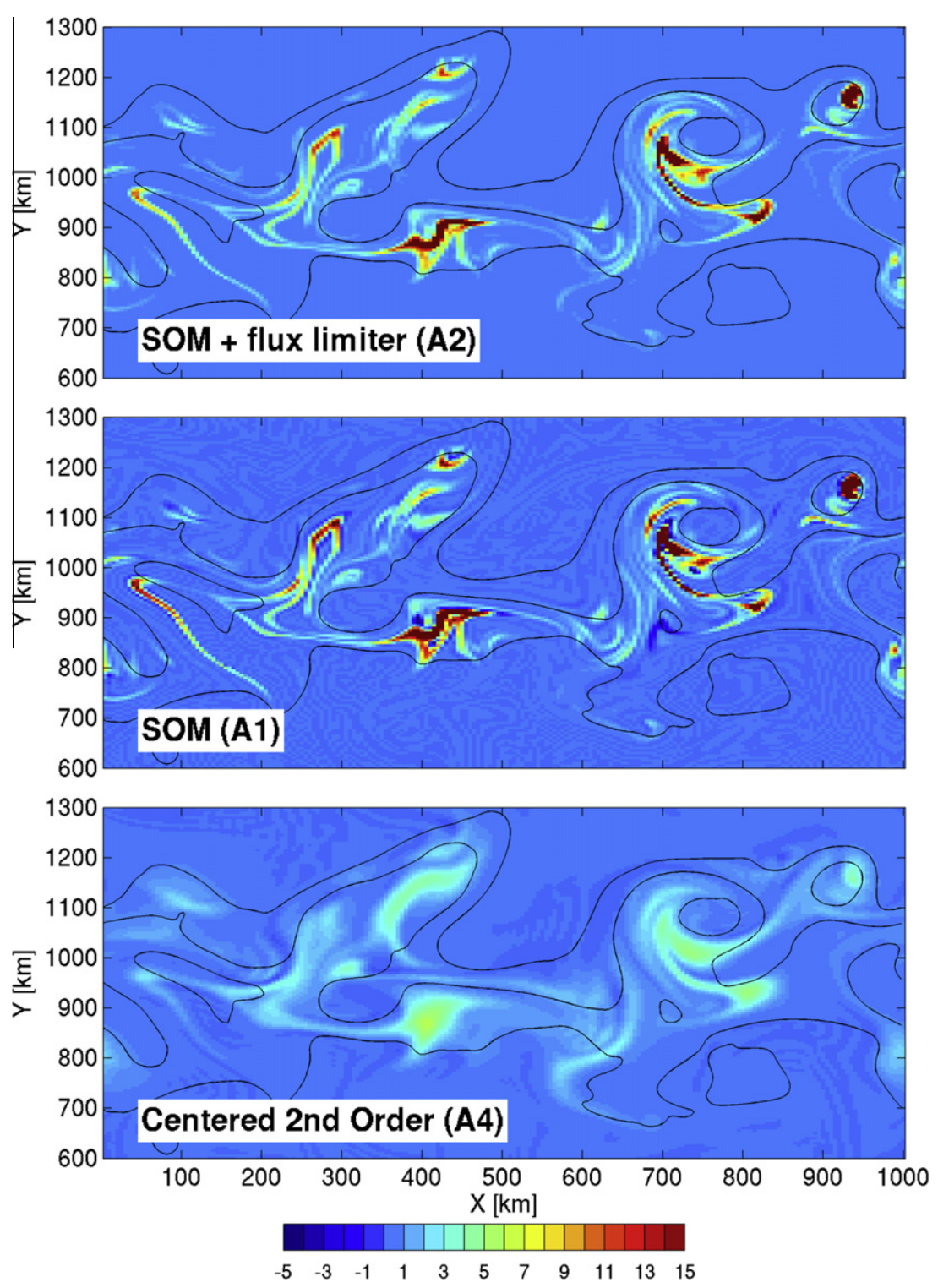

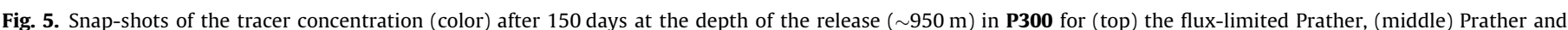

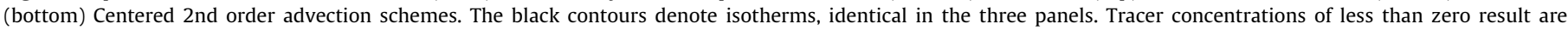

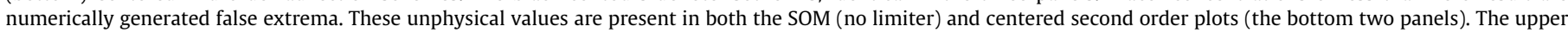
panel, SOM with simple limiter, has no values less than zero.

vertical variations in structure for P300 and $\mathbf{S 3 0 0}$ GPH00 diagnosed diffusivities are similar with elevated values near the surface, at "mid-depth" densities and for highest densities (lowest $z^{*}$ ).

A full analysis of elevated spurious mixing toward lower density levels (close to $z^{*}=0$ ) is outside the scope of this paper - our focus is on adiabatic advection in the ocean interior rather than the equally important (but different) challenge of how to represent flows and mixing in boundary regions. There are several factors that could contribute to the elevated values seen in our numerical experiments. Firstly, in the experiments analyzed there is a bottom friction term that produces a bottom Ekman layer. It is possible that vertical transports arising from Ekman convergence and divergence at the bottom of the model lead to enhanced mixing. Another source of apparent mixing could arise from the form of the GPHO0 diagnostic. The effective diapycnal mixing diagnostic models the creation and destruction of water masses as fluxes between "adjacent" water masses in density space. As discussed in GPHO0, interpreting this model for a discrete set of density classes requires care. For example a solution where there is mixing over a sharp gradient between two non-adjacent density classes is represented as elevated mixing across all the intermediate density classes - even though less formally it might be thought of as mixing just between the two non-adjacent classes. This effect may be contributing to the enhanced diagnosed mixing within dense water masses.

A second analysis using a passive tracer forms a virtual analog of deliberate tracer release field experiments. Fig. 5 shows snapshots of the passive tracer 150 days after release for three of the advection schemes (A1, A2 and A4) enumerated in Section 2.4. The flow field is that of $\mathbf{P 3 0 0}$ in the 3 cases. It is clear that the SOM scheme (flux limited or not - shown in the upper two panels) preserves the fine scale stirring of the tracer field, unlike the centered second order scheme. The filaments shown here resemble those detected by Ledwell et al. (1993) during the NATRE field experiment (see their Fig. 1). Reconstructed vertical profiles at 150 days are shown in Fig. 6 (left panel) for the same set of advec- 

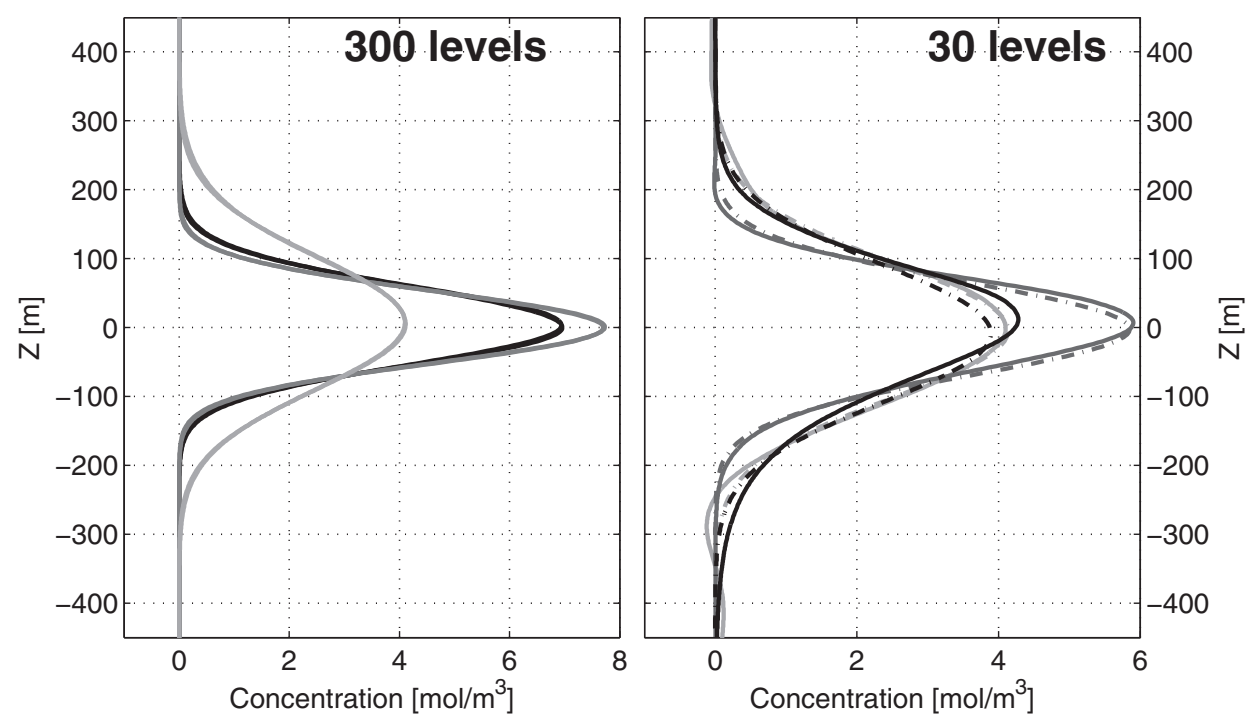

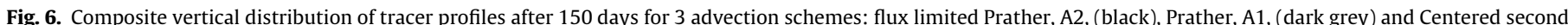

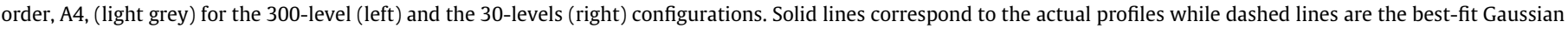
curves. The solid and dashed lines overlay one another in the left panel.

tion schemes. At this vertical resolution, the profiles are nearly exactly Gaussian. These are rather direct analogs of the vertical tracer spreading map in Ledwell et al. (1998), making them useful for connecting to observational results. (Note however that the initial tracer spreading in the vertical is $50 \mathrm{~m}$ in the model compared to about a meter in field experiments, due to finite vertical grid spacing in the numerical model.) Quantitative estimates of diapycnal mixing are derived from fitting the curves in Fig. 6 to a Gaussian. The evolution of the squared half-width of the fitted Gaussian is shown in Fig. 7. After some initial adjustment, $\sigma^{2}(t)$ grows linearly with time in all cases.

Best-fit linear curves for $t \geqslant 100$ days are shown by thin red lines on figure Fig. 7. The estimated effective diffusivity for the SOM scheme without a flux limiter is $\kappa_{e f f}^{\mathrm{L}}=0.005 \times 10^{-5} \mathrm{~m}^{2} \mathrm{~s}^{-1}$. This is lower than the GPHOO estimate for the target isotherm (compare the green diamond and black lines in Fig. 4), but is consistent with interior diffusivities for non-outcropping isotherms. Indeed, the passive tracer does not extend into the surface layers and samples only the ocean interior. This reinforces our interpretation that the somewhat elevated $\kappa_{\text {eff }}^{\mathrm{G}}$ above $z^{*}=1500 \mathrm{~m}$ in Fig. 4 is due to the stronger small scale cascade near the surface.

Also shown in the Fig. 4 are the tracer release diagnosed diffusivities, $\kappa_{\mathrm{eff}}^{L}$, for the SOM with limiter and 7 th order advection scheme advection schemes (A2 and A8 respectively in Table 2). The remaining tracer schemes have been tried and are illustrated in Figs. 5-7. All results are summarized in Table 2. Interestingly, the flux limited SOM scheme has reasonable values of spurious mixing. This is significant even though the limiter used only ensures non-negative values. For the tracer experiment the tracer minima is initially zero everywhere, except at the injection location so this limiter is active. The result is illustrated in Fig. 5 (top) where the tracer field is positive everywhere. In the unlimited SOM case (middle), a couple of under-shoots are noticeable (due to tracer release imposed at the grid-point scale). A further study using more sophisticated limiters (such as in Prather et al. (2008), Morales Maqueda and Holloway (2006)) would be a very valuable next step in this area. We expect the results from such a study would likely show a diffusivity close to the simple limiter employed in this study - as discussed in Prather et al. (2008). All other advection schemes result in larger effective diffusivities to varying degrees.
We conclude that the GPHOO and passive tracer estimates show consistent behaviors, revealing very small spurious diapycnal mixing in the ocean interior with the SOM scheme for this model. In an extra test an explicit vertical diffusivity $\kappa_{v}=10^{-5} \mathrm{~m}^{2} \mathrm{~s}^{-1}$ was added to the unlimited SOM advection test, A1. In this additional experiment the Ledwell method computation of mixing gave an estimate of $0.95 \times 10^{-5} \mathrm{~m}^{2} \mathrm{~s}^{-1}$. This bolsters the notion that the Ledwell approach for this numerical configuration can yield valid estimates of diffusivity. Good correspondence between an explicit $\kappa_{v}$ and a tracer released diagnosed estimate was confirmed for values of explicit $\kappa_{v}$ ranging from $10^{-6}$ to $10^{-4} \mathrm{~m}^{2} \mathrm{~s}^{-1}$.

\subsection{0 level experiments}

For the 30-level case, 5 runs with different advection schemes on temperature were spun-up (Table 1). Results of the spin-down effective diffusivity estimations are shown in Fig. 8. Results from tracer release experiments with flow from P30 are summarized in Table 2. The 4 tracer release experiments whose advection schemes correspond to the spin-down experiments are also reported on Fig. 8 for comparison of the 2 methods. Examples of the time-evolution of $\sigma^{2}(t)$ are shown in Fig. 9. These are taken from the Gaussian curves in the right panel of Fig. 6. In all the tracer release tests with 30 levels an ensemble of releases was generated with tracer injected at slightly different locations. This ensemble provides a crude means to estimate the sensitivity of the estimated diffusivity to injection details. The right panel in Fig. 10 shows $\sigma^{2}(t)$ for all the ensemble members for the A1, A2, A3 and A4 30 level experiments.

A 30 level side experiment, P30_HR, reinforces the argument that elevated surface values are more physical than numerical. For this experiment the model horizontal resolution was doubled to $2.5 \mathrm{~km}$ and a solution spun up to statistical equilibrium. Then numerical diffusivities were diagnosed from a spin-down experiment. This result is shown in Fig. 10. The profile is very similar to the base set of $5 \mathrm{~km}$ resolution experiments. If the results were dominated by pure numerical artifacts, the increased straining in the increased resolution case would likely lead to elevated effective diffusivity. This is not observed in this experiment.

Again, the unlimited SOM experiment $\mathbf{P 3 0}$ has the lowest values of spurious diapycnal mixing, below estimates from tracer release 


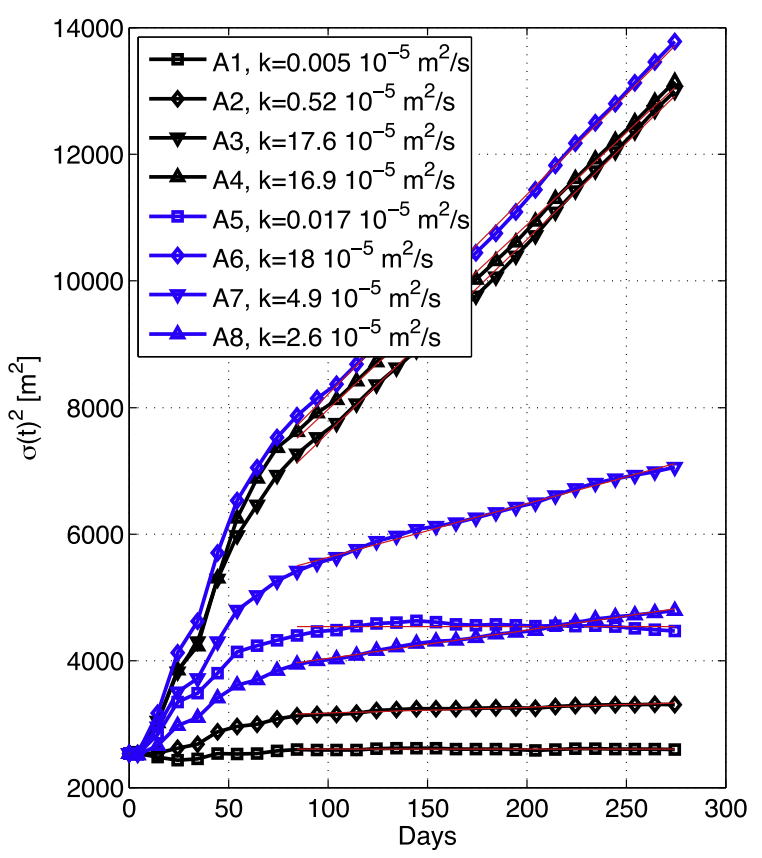

Fig. 7. Time evolution of the squared half-width $\sigma(t)^{2}$ of the fitted Gaussian to the reconstructed profile. The passive tracers are advected by the flow from P300. Diffusivity estimates in Table 2 are computed from the slopes of these lines (shown in red) - excluding an initial transient. (For interpretation of the references in color in this figure legend, the reader is referred to the web version of this article.)

field experiments. Values from the tracer release experiment for the SOM scheme are about 3 times those from the spin-down experiment. The tracer release experiment is associated with large gradients at the grid-scale, enhancing the effective diffusivities. (In fact, the initial tracer distribution on the 30 level vertical grid is nearly a "top-hat" with all the tracer concentrated at one level. Such unphysical distributions do not arise in the temperature field). The spin-down experiment probably gives a better estimation of tracer diapycnal mixing relevant to climate modeling problems. However, tracer release tests provide a more direct link to field studies and so are useful to highlight the discrepancy between simulation behavior and the real ocean. A calibration experiment with the SOM (no limiter) scheme, in which a background explicit vertical diffusion of $\kappa_{v}=1 \times 10^{-5} \mathrm{~m}^{2} \mathrm{~s}^{-1}$ was included, gave increased estimated mixing of $1.75 \pm 0.24 \times 10^{-5} \mathrm{~m}^{2} \mathrm{~s}^{-1}$ (compared to $0.9 \pm 0.2 \mathrm{~m}^{2} \mathrm{~s}^{-1}$ ). The SOM with limiter spin-down experiment

\section{Table 2}

Effective diffusivities estimated from the tracer release experiments. The first and second columns indicate the advection scheme used. All diffusivities are in $10^{-5} \mathrm{~m}^{2} \mathrm{~s}^{-1}$. The third and fourth columns are the diffusivities derived using flow fields from the no limiter SOM advection experiments with 30 levels, P30, and 300 levels, P300. For experiments $\mathbf{A} \mathbf{3}$ and $\mathbf{A 4}$ explicit horizontal and vertical diffusivities of $30 \mathrm{~m}^{2} \mathrm{~s}^{-1}$ and $10^{-5} \mathrm{~m}^{2} \mathrm{~s}^{-1}$ respectively are applied.

\begin{tabular}{|c|c|c|c|}
\hline $\begin{array}{l}\text { Advection } \\
\text { code }\end{array}$ & Description & $\begin{array}{l}\text { Diffusivity } \\
\text { (P30 flow) }\end{array}$ & $\begin{array}{l}\text { Diffusivity } \\
\text { (P300 } \\
\text { flow) }\end{array}$ \\
\hline A1 & SOM (no limiter) & $0.93 \pm 0.2$ & 0.005 \\
\hline A2 & SOM (simple limiter) & $3.8 \pm 2$ & 0.52 \\
\hline A3 & SOM (no limiter) with diffusion & $18 \pm 2$ & 17.6 \\
\hline A4 & Centered 2 nd order with diffusion & $19 \pm 5$ & 16.9 \\
\hline A5 & $\begin{array}{l}\text { 2nd order with "Superbee" Roe } \\
\text { (1985) limiter. }\end{array}$ & $11 \pm 5$ & 0.017 \\
\hline A6 & $\begin{array}{l}\text { 3rd order direct space time } \\
\text { Hundsdorfer and Verwer (2003). }\end{array}$ & $32 \pm 4$ & 18 \\
\hline A7 & $\begin{array}{l}\text { 3rd order direct space time with } \\
\text { Sweby limiter (Sweby, 1984). }\end{array}$ & $24 \pm 4$ & 4.9 \\
\hline A8 & $\begin{array}{l}\text { 7th order one-step method, with } \\
\text { monotonicity preserving limiter } \\
\text { (Daru and Tenaud, 2004). }\end{array}$ & $12 \pm 3$ & 2.6 \\
\hline
\end{tabular}

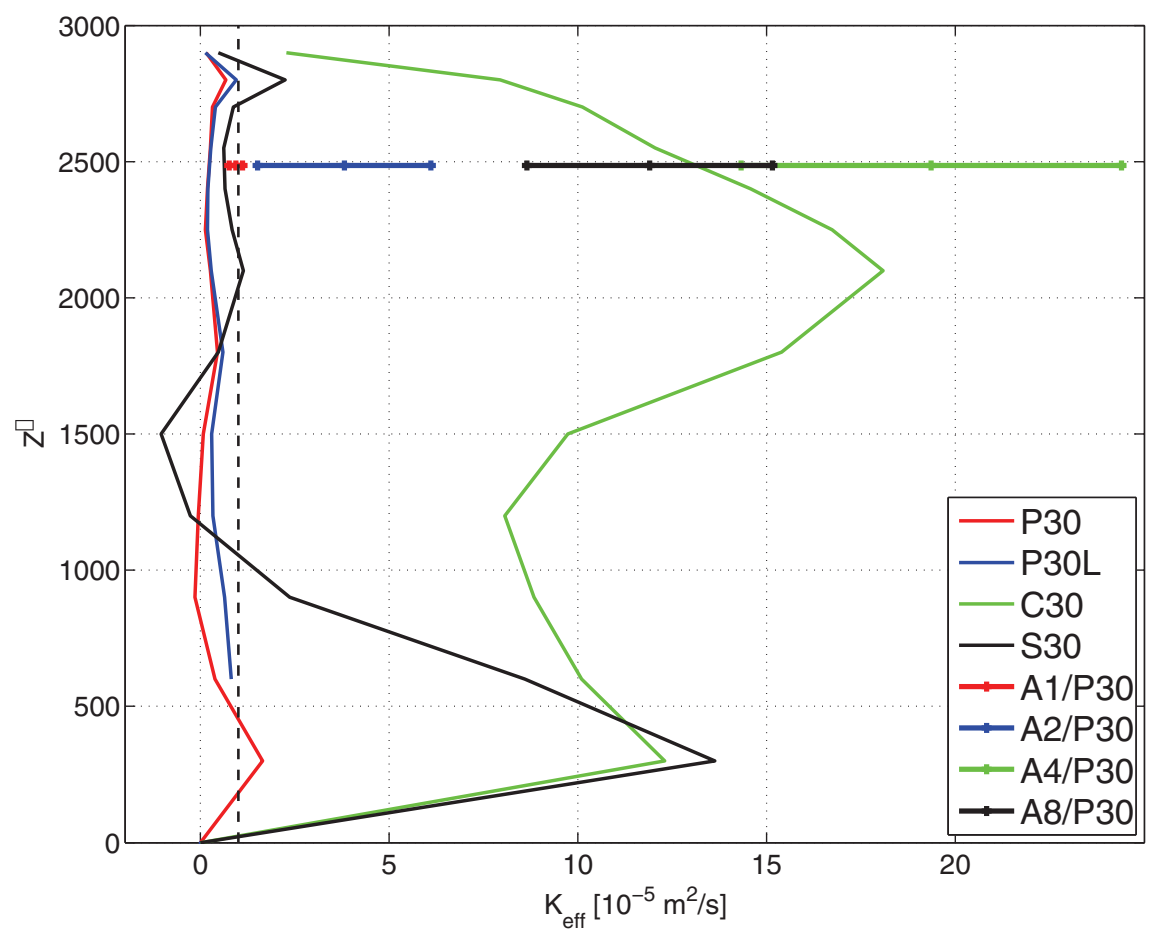

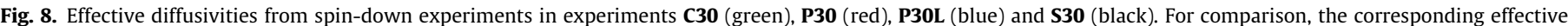

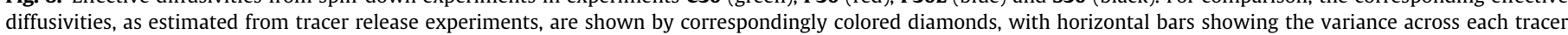

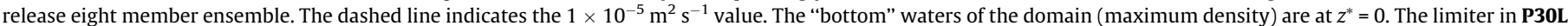

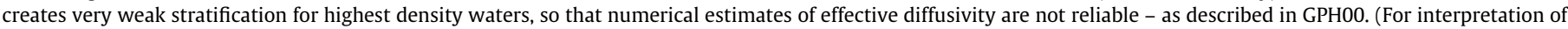
the references in color in this figure legend, the reader is referred to the web version of this article.) 

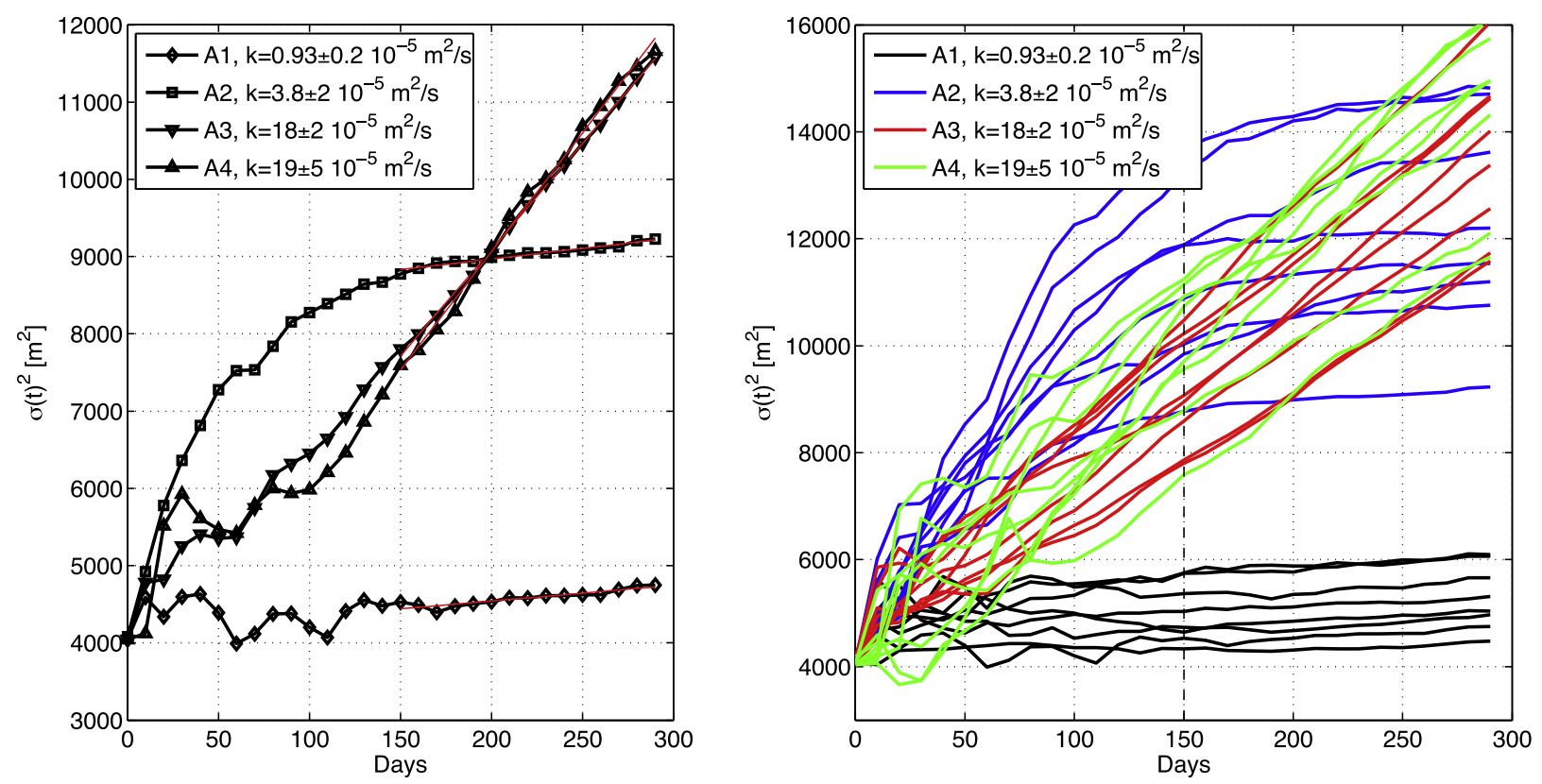

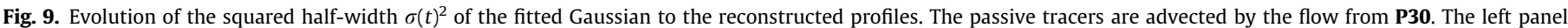

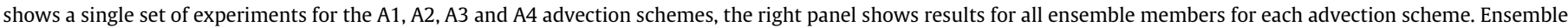

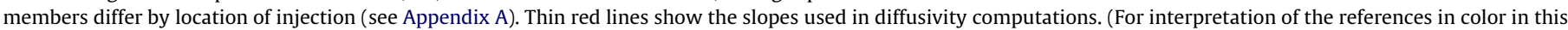
figure legend, the reader is referred to the web version of this article.)

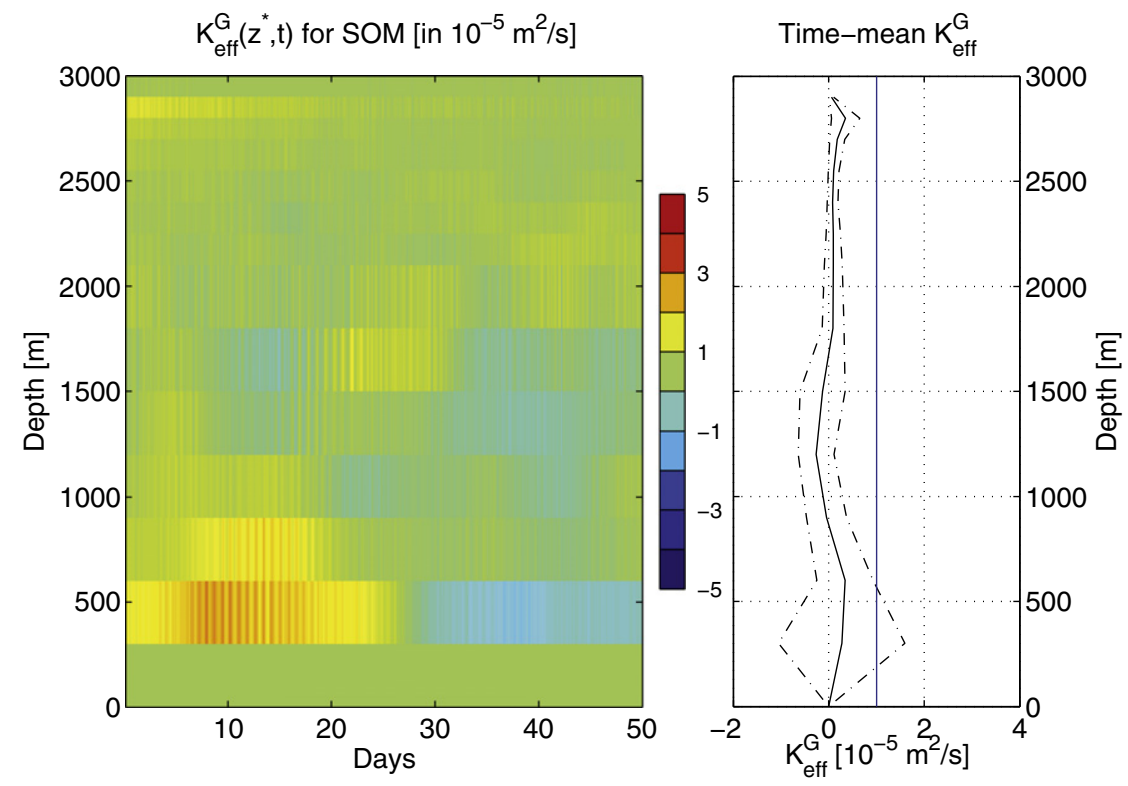

Fig. 10. Effective diapyncnal diffusivity for $2.5 \mathrm{~km}$ horizontally enhanced resolution experiment P30_HR configuration.

(P30L) is the only other scheme that produces diapycnal mixing below $1 \times 10^{-5} \mathrm{~m}^{2} \mathrm{~s}^{-1}$. This suggests that, for this experiment, this particular limiter provides an effective way to ensure positive definite behavior, without inducing excessive diapycnal mixing.

As expected, the centered second order scheme results in large effective diapycnal mixing, considerably exceeding $10^{-4} \mathrm{~m}^{2} \mathrm{~s}^{-1}$. This is due to the explicit horizontal and vertical diffusion required for this scheme to obtain an acceptable temperature distribution. Note, however that these diffusion values are in fact too low for the sharp gradients of the released tracer as slightly negative tracer values are seen in the reconstructed profile (Fig. 8). The 7th order scheme effective diffusivities are satisfying at high $z^{*}$ (high temper- ature). There are however very large $\kappa_{\text {eff }}^{G}$ at cold temperature and significant negative values at intermediate temperature $\left(z^{*} \sim 1500 \mathrm{~m}\right)$.

As seen in Table 2, all other advection schemes perform poorly, even when considering that the 30 level tracer release appears to overestimate the effective diffusivities at coarse vertical resolution.

\subsection{Residual-mean circulations}

A final, and perhaps most oceanographically significant, measure of the fidelity of the numerical model is shown in the residual circulation plots in Figs. 11 and 12. Where the methods of GPHOO 

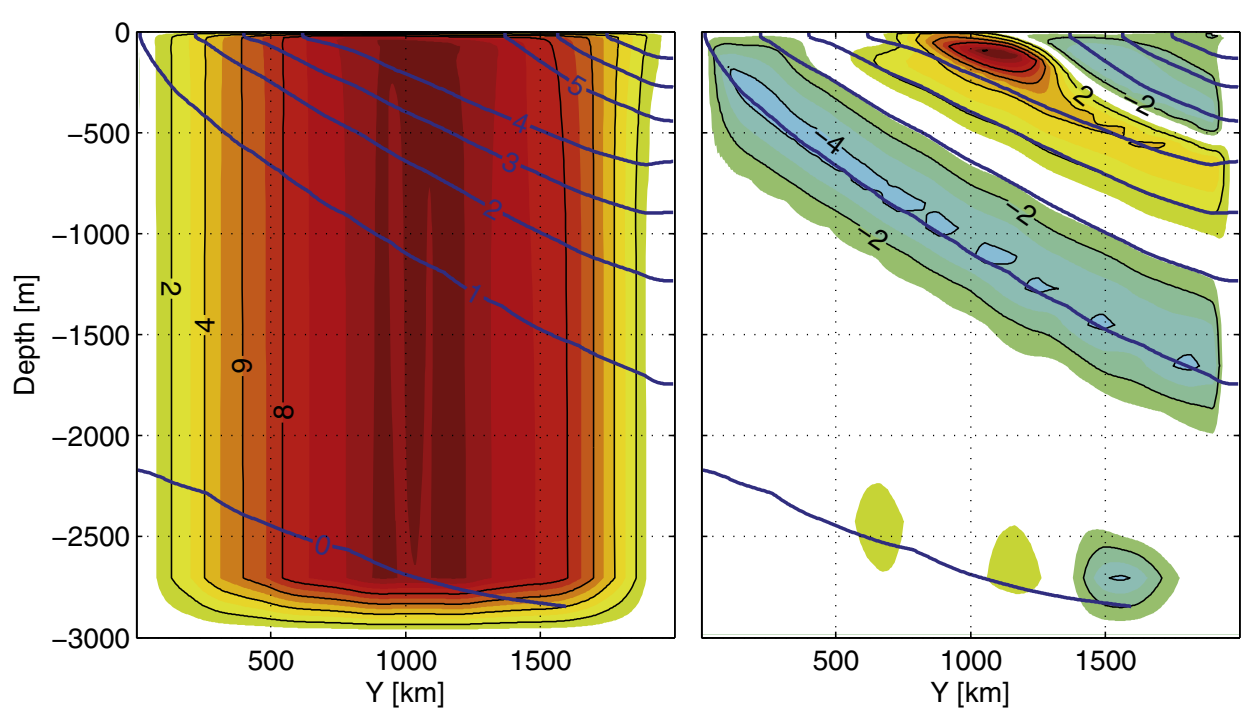

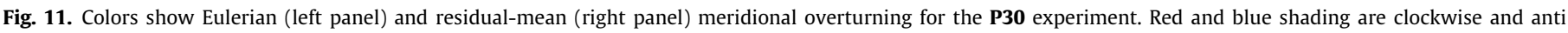

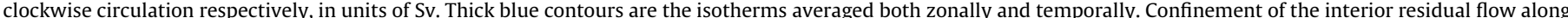

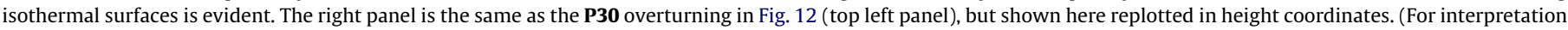
of the references in color in this figure legend, the reader is referred to the web version of this article.)
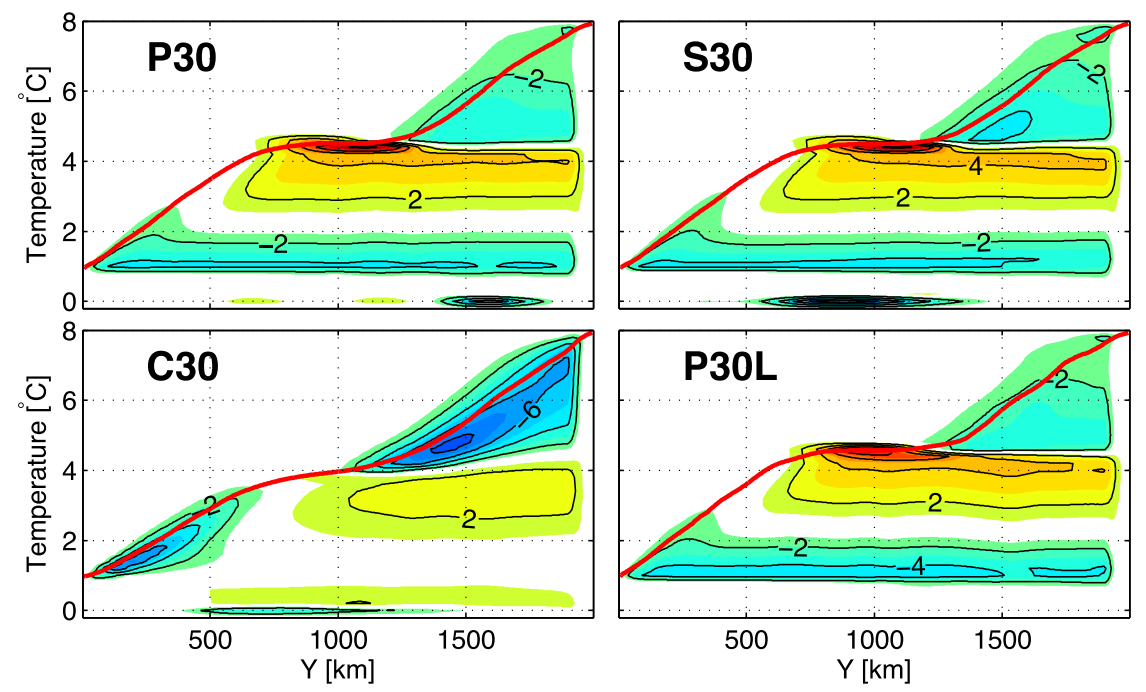

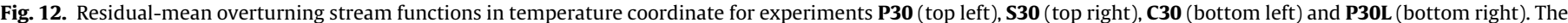

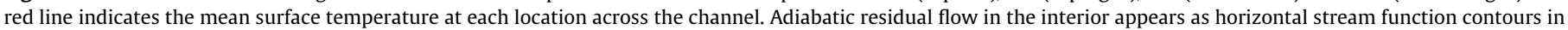
this coordinate system. (For interpretation of the references in color in this figure legend, the reader is referred to the web version of this article.)

and Ledwell like tracer release provide quantitative metrics for mixing, the residual circulation provides a gross measure of the significant role of spurious mixing in overall circulation patterns. These plots show the residual transport in different coordinates for the four different 30 level model configurations (P30, P30L, C30, S30). Fig. 11 shows both the Eulerian overturning (left panel) and the residual circulation (right panel) in height coordinates for the P30 experiment. Both have mean isotherms overlaid. The alignment of the residual circulation with isothermal/isopycnal surfaces is clear in the right panel. Fig. 12 shows residual circulation in temperature coordinates for the P30, P30L, S30 and C30 integrations. The residual-mean circulation stream lines are horizontal for these experiments, that is they lie along isopycnal surfaces, in the domain interior and diapycnal processes are confined to boundary regions. In the $\mathbf{C 3 0}$ experiments, the residual circulation crosses isopycnal surfaces in the interior as well as in boundary regions. Comparison of the various panels in Fig. 12 makes obvious the impact of the spurious mixing on the large scale circulation of the ocean. Such differences are expected to drastically influence exchange between the surface and the interior ocean, rates of heat and carbon sequestration, amongst many other things.

\section{Conclusion}

Exploring the impact of numerical schemes on simulations is a time honored exercise e.g. Rood (1987), Strang (1968), and Griffies et al. (2000). Here, by examining the implications of different tracer advection schemes on diapycnal mixing in a reentrant channel model, we have identified SOM as having desirable properties that appear to go a long way toward resolving a key long-standing conundrum facing $\mathrm{z}$-coordinate numerical ocean simulations in eddying regimes. 
We have explored several virtual analogs to the NATRE and DIMES field experiments. Spurious diapycnal mixing when using the SOM scheme with 300 levels is below observed levels and the interior residual circulation aligns with isopycnal surfaces. A computationally less expensive 30 level configuration, also using the SOM scheme, exhibits many of the properties of the 300 level calculation. It too has acceptably small numerically induced diapycnal mixing (even when employing simple flux limiting) and again has a residual circulation coincident with isopycnals in the interior. The other schemes examined all exhibit unacceptably high values of diapycnal mixing and, as a result, the residual circulation is no longer robustly aligned with isopycnals in the model interior.

Our results have particular significance for ongoing activities aimed at modeling the ocean on climate time scales at resolutions that fully resolve the eddy field (e.g. 1-5 km horizontal resolution). Such models no longer parameterize geostrophic eddy processes and so accurate representation of eddy impacts is important. In particular, numerical schemes that overstate interior diapycnal mixing will tend to be systematically biased with respect to the real ocean. Uptake of heat and carbon into the ocean on climate timescales would likely be sensitive to the advection schemes examined. Key properties associated with water mass formation and subduction may also be sensitive to the advection scheme, as would rates of draw down of atmospheric gases, affecting patterns of ocean biology.

Abernathey et al. (2011) have shown that the SOM scheme is practical for ambitious modeling studies. There are extra costs in SOM due to increased memory footprint. For every 3 dimensional field SOM requires the storage of 10 coefficients that characterize the distribution of the field within a grid cell (as discussed in the Appendix). However, these additional coefficients lead to a scheme in which the discrete spatial stencil is relatively compact (in comparison to schemes such as $\mathbf{S 3 0}$ ) so that the computational cost of SOM is not overwhelming. For example, in Hofmann and Morales Maqueda (2006) SOM accounts for $10-20 \%$ of the overall compute time. In Abernathey et al. (2011) SOM evaluation represents a 20\% compute time overhead relative to equivalent 7 th order one-step method (Daru and Tenaud, 2004) experiments. Overall we conclude that even though SOM is somewhat computationally demanding the obvious benefits are potentially significant, suggesting that the z-coordinate community should seriously consider SOM approaches for eddying scenarios.

Future work that would be beneficial to this direction includes examining the utility of using a SOM scheme for active tracers while maybe using a less expensive or alternate approach for biogeochemical properties and other quantities typically assumed to be dynamically inert. The limiter applied to the SOM formulation in this work is a relatively crude scheme. Further work should also look at more recent limiters such as in Prather et al. (2008) and Morales Maqueda and Holloway (2006). Analysis in Prather et al. (2008) suggests that the implied diffusivity could be elevated, but not dramatically, for a fully monotonic limiter. Finally we note that we have not exhaustively explored every advection scheme or diagnostic approach for determining diffusivities. Studies of schemes such as MPDATA and PPM would be enlightening as would studies using alternate methods, such as Burchard and Rennau (2008) to estimate numerical mixing and studies of the role of momentum equation formulation (Ilicak et al., in press) in overall solution character.

\section{Acknowledgements}

We would like to thank our reviewers, Stephen Griffies, Mehmet Iliack and two others, for their useful and informative suggestions and advice. Their input helped significantly.

\section{Appendix A}

Here we present some technical details of the computations we have employed. In Sections A.1 and A.2, we briefly describe details of the methods used to estimate the effective diapycnal diffusivities, their limitations and associated uncertainties. Related discussion, for a one-dimensional problem, can be found in Getzlaff et al. (2010). Finally, in Section A.3 we examine the SOM sub-grid coefficients.

\section{A.1. The Ledwell et al. method}

When mimicking Ledwell et al. tracer release experiments (see description in Section 2.4) in an ocean model, two issues arise in relation to the initialization of the passive tracer: (1) finding an initial tracer distribution consistent with the fully developed turbulent flow and (2) avoiding large tracer gradients at the grid scale which result in large overshoots. The first issue is easily tackled by letting the tracer adjust to the flow for a period of time before monitoring its behavior and computing the effective diffusivity. To avoid large gradients at the grid scale, the tracer field is initialized with a $3 \mathrm{~d}$ Gaussian shape with $50 \mathrm{~m}$ vertical and a $5 \mathrm{~km}$ horizontal half-width. The tracer has a maximum value of one and is released at a depth of about $950 \mathrm{~m}$ in the 300 level experiments and at about $500 \mathrm{~m}$ in the 30 level experiments. The $50 \mathrm{~m}$ vertical scale is well resolved by the $10 \mathrm{~m}$ vertical spacing of the 300 -level configuration, less so in the 30-level configuration where overshoots are observed. However, this initialization pattern, leaves ample time before the tracer is transported into the surface mixed layer and gradients are mixed away.

Another limitation of the method is due to the interpolation required to reconstruct a single profile. This results in a small source/ sink of tracer. Note however that in the model the reconstructed profile recovers the total tracer amount within 1\% (much better than in field experiments). Finally, the reconstructed profile is fitted to a Gaussian and the Gaussian peaks are aligned. As illustrated in Fig. 6 (left), the reconstructed profiles in the 300-level config uration are nearly indistinguishable from Gaussian curves for all advection schemes. In the 30-level configuration, this is less accurate (see in particular the negative values below the peak for the centered second order scheme). The Gaussian fit uses part of the profile with values greater than $10 \%$ of the maximum. This choice has almost no effect in the 300-level case, but sensitivity tests show that in the 30 -level case there is a $\pm 10 \%$ uncertainty attached to this choice. Because results at 300 levels are very robust (and consistent with those of the GPHO0 method), one single tracer release is used for each advection test. With 30 levels however, 8 tracers releases (at the same depth and time but $125 \mathrm{~km}$ apart in the zonal direction) are used for each advection scheme to quantify uncertainties associated with the method.

A final limitation is the impact of vertical spreading of isopycnals. The spreading of an isopycnal containing tracer will induced a broadening of the Gaussian, without requiring diapycnal mixing (see discussion in Getzlaff et al. (2010) for example). In general the effects listed here will combine to produce an estimated diapycnal mixing that is greater than the GPHOO estimate, but that can still discriminate between advection schemes.

\section{A.2. The GPHOO method}

In practice, the first step of the GPHO0 method (Fig. 3) requires one to sort the $N_{x} \times N_{y} \times N_{z}$ grid points of the computational domain according to their (increasing) temperature, keeping track of the volume associated with each grid-point $V_{i}$. In the $z^{*}$ space, each temperature point is then attributed a vertical spacing 


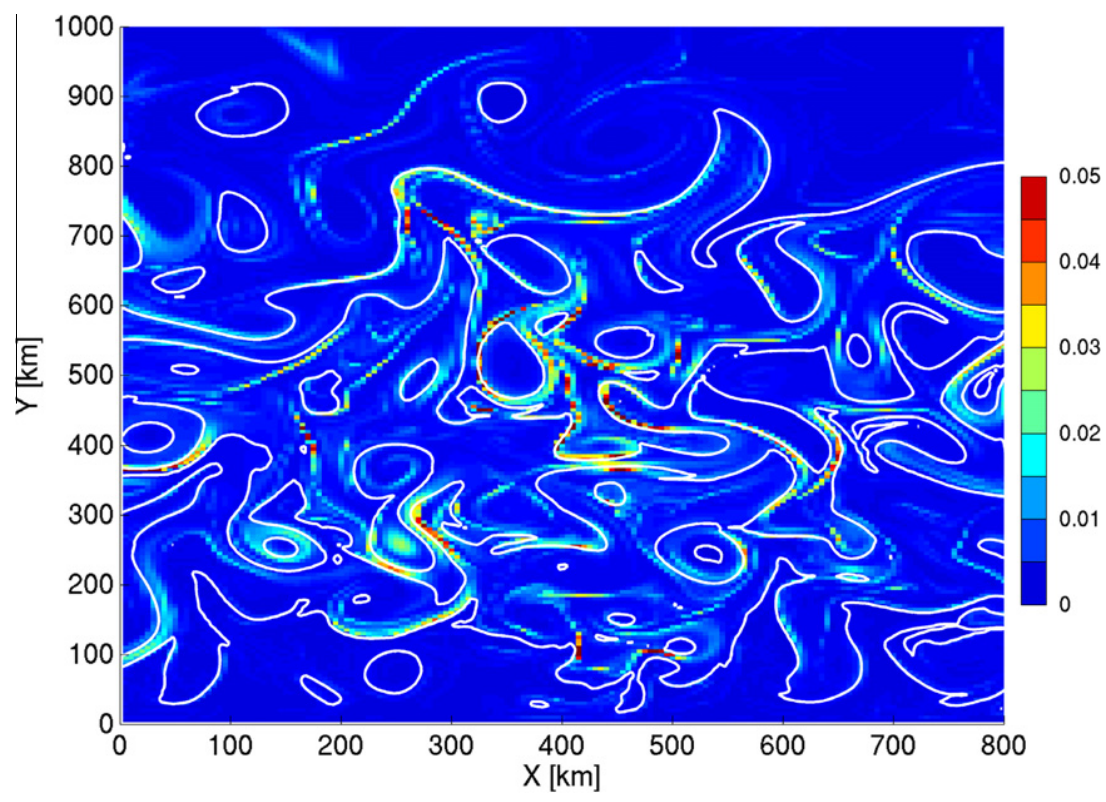

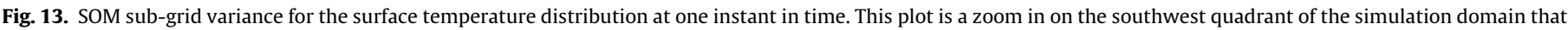

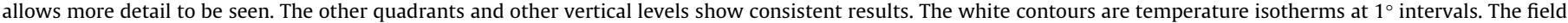
shown is taken from the $\mathbf{P 3 0 0}$ experiment.

$d z^{*}=V_{i} /\left(L_{x} L_{y}\right)$ where $L_{x} L_{y}$ is the horizontal area of the channel. By construction, the sorted $\theta\left(z^{*}, t\right)$ profile extends from $z^{*}=0$ (coldest grid-point) to $z^{*}=3000 \mathrm{~m}$ (warmest grid-point). Note that the discretization in $z^{*}$ thus obtained is a function of the model state and varies in time. Therefore, the sorted $\theta^{*}$ profiles need to be interpolated to a reference vertical grid in $z^{*}$ for solving Eq. (3). As noted by GPH00, the interpolated $\theta^{*}$ still retains small scale structures which can lead to noise when solving for $\kappa_{\text {eff }}^{G}$ from Eq. (3). Following their procedure (in which interpolation is performed with a monotonic, cubic scheme), the reference vertical $z^{*}$ grid is chosen to be similar to the physical $z$ spacing of the channel and the interpolated profiles are smoothed with a 1-2-1 filter before solving Eq. (3). Overall the approach we take follows the method outlined in GPHO0, and as such has the limitations noted in that paper.

\section{A.3. SOM sub-grid variance}

The SOM advection scheme utilizes 10 coefficients per grid cell to represent sub-grid spatial distribution of a single field. These coefficients are the magnitudes of moments that capture the subgrid variation of the field. Although, in this article, we are interested primarily in the bulk averages of a field over a single grid cell, it is nevertheless instructive to examine the moments that underly the advection procedure for the SOM schemes. One representation of the SOM sub-grid variance is shown in Fig. 13. This figure captures the variance of a field, $\phi$ (here temperature), that varies within a grid cell according to the SOM relation

$$
\begin{aligned}
\phi(x, y, z)= & a_{0}+a_{x} x+a_{x x} x^{2}+a_{y} y+a_{y y} y^{2}+a_{z} z+a_{z z} z^{2}+a_{x y} x y \\
& +a_{x z} x z+a_{y z} y z
\end{aligned}
$$

The variance term plotted is computed by subtracting squared mean of $\phi$ within each cell from the mean of $\phi$ within that cell squared

$$
\left(\frac{1}{V} \int_{0}^{Z} \int_{0}^{Y} \int_{0}^{X} \phi^{2} d x d y d z-\left\{\frac{1}{V} \int_{0}^{Z} \int_{0}^{Y} \int_{0}^{X} \phi d x d y d z\right\}^{2}\right)
$$

where $X, Y, Z$ are individual grid cell extents and $V$ is the cell volume. The figure shows, in a compact form, that the largest sub-grid variation occurs where the field being represented has sharp gradients. It shows that the magnitudes of the variations are consistent with the field being represented. For example, in Fig. 13, the variance has a maximum of $0.05{ }^{\circ} \mathrm{C}^{2}$ for a field, temperature, with bulk averages ranging between 0 and $8{ }^{\circ} \mathrm{C}$. Although not the subject of this paper (our diagnostics concern bulk averages for a grid cell), this plot suggest that the sub-grid variations in the SOM representation of a field do have some physical basis. The plots also show that in regions where the underlying field is smooth, the sub-grid variance is small - again consistent with a physical interpretation.

\section{References}

Abernathey, R., Marshall, J., Shuckburgh, E., Mazloff, M., 2010. Enhancement of mesoscale eddy stirring at steering levels in the southern ocean. J. Phys. Oceanogr. 40, 170-185.

Abernathey, R., Marshall, J., Ferreira, D., 2011. The southern ocean meridional overturning circulation dependence on wind strength. J. Phys. Oceanogr. 41, 2261-2278.

Andrews, D.G., McIntyre, M.E., 1976. Planetary waves in horizontal and vertical shear: The generalized eliassen-palm relation and the mean zonal acceleration. J. Atmos. Sci. 33, 2031-2048.

Burchard, H., Rennau, H., 2008. Comparative quantification of physically and numerically induced mixing in ocean models. Ocean Modell. 20, 293-311.

Daru, V., Tenaud, C., 2004. High order one-step monotonicity-preserving schemes for unsteady compressible flow calculations. J. Comput. Phys. 193, 563-594.

Garrett, C., Munk, W., 1972. Space-time scales of internal waves. Geophys. Astrophys. Fluid Dyn. 3, 225-264.

Getzlaff, J., Nurser, G., Oschlies, A., 2010. Diagnostics of diapycnal diffusivity in zlevel ocean models. Part i: 1-dimensional case studies. Ocean Modell. 35, 173186. doi:10.1016/j.ocemod.2010.07.004.

Griffies, S.M., Pacanowski, R.C., Hallberg, R.W., 2000. Spurious diapycnal mixing associated with advection in a z-coordinate ocean model. Mon. Wea. Rev. 128, 538-564.

Hofmann, M., Morales Maqueda, M.A., 2006. Performance of a second-order moments advection scheme in an ocean general circulation model. J. Geophys. Res. 111. doi:10.1029/2005JC003279.

Hundsdorfer, W., Verwer, J., 2003. Numerical Solution of Time-dependent Advection-diffusion-reaction Equations. Springer Verlag.

Ilicak, M., Adcroft, A., Griffies, S.M., Hallberg, R., in press. Spurious dianeutral mixing and the role of momentum closure. Ocean Modell. 
Large, W.G., McWilliams, J.C., Doney, S.C., 1994. Oceanic vertical mixing: A review and a model with a nonlocal boundary layer parameterization. Rev. Geophys. 32, 363-403.

Ledwell, J.R., Watson, A.J., Law, C., 1993. Evidence for slow mixing across the pycnocline from an open-ocean tracer-release experiment. Nature 364, 701703.

Ledwell, J.R., Watson, A.J., Law, C.S., 1998. Mixing of a tracer in the pycnocline. J. Geophys. Res. 103, 21499.

Ledwell, J.R., St. Laurent, L.C., Girton, J.B., Toole, J.M., 2011. Diapycnal mixing in the Antarctic circumpolar current. J. Phys. Oceanogr.. doi:10.1175/2010JPO4557.1 <http://journals.ametsoc.org/doi/abs/10.1175/2010JPO4557.1>

Marshall, J., Radko, T., 2003. Residual mean solutions for the antarctic circumpolar current and its associated overturning circulation. J. Phys. Oceanogr. 33, 23412354.

Marshall, J., Adcroft, A., Hill, C., Perelman, L., Heisey, C., 1997a. A finite-volume, incompressible Navier Stokes model for studies of the ocean on parallel computers. J. Geophys. Res. 102, 5753-5766.

Marshall, J., Hill, C., Perelman, L., Adcroft, A., 1997b. Hydrostatic, quasi-hydrostatic, and nonhydrostatic ocean modeling. J. Geophys. Res. 102, 5733-5752.

McIntosh, P.C., McDougall, T.J., 1996. Isopycnal averaging and the residual mean circulations. J. Phys. Oceanogr. 26, 1655-1661.

Merryfield, W., Holloway, G., 2003. Application of an accurate advection algorithm to sea-ice modelling. Ocean Modell. 5, 1-15.

Morales Maqueda, M.A., Holloway, G., 2006. Second-order moment advection scheme applied to arctic ocean simulation. Ocean Modell. 14, 197-221.

Phillips, H., Rintoul, S., 2000. Eddy variability and energetics from direct current measurements in the antarctic circumpolar current south of australia. J. Phys. Oceanogr. 30, 3050-3076.
Polzin, K.L., Toole, J.M., Ledwell, J.R., Schmitt, R.W., 1997. Spatial variability of turbulent mixing in the abyssal ocean. Science 276, 93-96.

Prather, M., 1986. Numerical advection by conservation of second-order moments. J. Geophys. Res. 91, 6671-6681.

Prather, M., Zhu, X., Strahan, S., Steenrod, S., Rodriguez, J., 2008. Quantifying errors in trace species transport modeling. Proc. Nat. Acad. Sci. 105, 19617.

Roe, P., 1985. Some contributions to the modelling of discontinuous flows. Largescale Comput. Fluid Mech. 1, 163-193.

Rood, R., 1987. Numerical advection algorithms and their role in atmospheric transport and chemistry models. Rev. Geophys. 25, 71-100.

Russell, G., Lerner, J., 1981. A new finite-differencing scheme for the tracer transport equation. J. Appl. Meteorol. 20, 1483-1498.

Strang, G., 1968. On the construction and comparison of difference schemes. SIAM J. Numer. Anal. 5, 506-517.

Sweby, P., 1984. High resolution schemes using flux limiters for hyperbolic conservation laws. SIAM J. Numer. Anal. 21, 995-1011.

Tatebe, H., Hasumi, H., 2010. Formation mechanism of the pacific equatorial thermocline revealed by a general circulation model with a high accuracy tracer advection scheme. Ocean Modell. 35, 245-252. doi:10.1016 j.ocemod.2010.07.011 <http://www.sciencedirect.com/science/article/pii/ S1463500310001125>.

Treguier, A., Held, I., Larichev, V., 1997. Parameterization of quasigeostrophic eddies in primitive equation ocean models. J. Phys. Oceanogr. 27, 567-580. 